\title{
Association of HLA-C*06 with Psoriasis in a Tertiary Care Hospital
}

\author{
Ashwin Anandan ${ }^{1}$, Ravindraprasad Thokala ${ }^{2}$, Krishnamoorthy \\ Radhakrishnan ${ }^{3}$, Vinod Kumar Panicker ${ }^{4}$, Murugan Sundaram ${ }^{5}$
}

\begin{abstract}
${ }^{1}$ Department of Transfusion Medicine, Sri Ramachandra Medical College and Research Institute, Chennai, Tamilnadu, India. ${ }^{2}$ Department of Transfusion Medicine, Sri Ramachandra Medical College and Research Institute, Chennai, Tamilnadu, India. ${ }^{3}$ Department of Transfusion Medicine, Sri Ramachandra Medical College and Research Institute, Chennai, Tamilnadu, India. ${ }^{4}$ Department of Transfusion Medicine, Sri Ramachandra Medical College and Research Institute, Chennai, Tamilnadu, India. ${ }^{5}$ Department of Dermatology, Sri Ramachandra Medical College and Research Institute, Chennai, Tamilnadu, India.
\end{abstract}

\section{ABSTRACT}

\section{BACKGROUND}

Prevalence of psoriasis is $1-3 \%$ in India. HLA-C*06 has been shown to be strongly associated with psoriasis in different ethnic populations. This study was carried out to determine the association of HLA-C in psoriasis patients in a south Indian ethnic population.

\section{METHODS}

A total of 200 samples were included in the study. In all, 100 psoriasis patients and 100 healthy controls were studied. HLA-C typing was done by PCR-SSP method. Results were analysed statistically using open epi software ( 2 X 2 table). The Odds ratio (OR), p (probability) value, and 95\% confidence interval were the statistical tests applied and analysed.

\section{RESULTS}

A total of 14 different HLA-C alleles were identified in both 100 cases and 100 controls. Among the 14 different HLA-C alleles, the alleles which were found to be strongly associated with psoriasis which were statistically significant were both HLA$\mathrm{C}^{*} 06$ and HLA-C*07. HLA-C*06 was found to be present in $52 \%$ of the patients and HLA-C* 07 was found to be present in $33 \%$ of the patients. HLA-C*06 was found to be strongly associated with the disease in $52 \%$ of the patients.

\section{CONCLUSIONS}

This study confirms HLA-C*06 association with psoriasis which is in concordance with other previous studies.

\section{KEY WORDS}

Human Leucocyte Antigen [HLA], Polymerase Chain Reaction-Sequence Specific Primers [PCR-SSP], Psoriasis, Odds Ratio [OR]
Corresponding Author:

Dr. Ravindraprasad Thokala, Department of Transfusion Medicine,

Sri Ramachandra Medical College and Research Institute, Porur, Chennai-600116, Tamilnadu, India.

E-mail:drravi1212@gmail.com

DOI: $10.14260 / j e m d s / 2020 / 341$

Financial or Other Competing Interests: None.

How to Cite This Article:

Anandan A, Thokala R, Radhakrishnan K, et al. Association of HLA-C*06 with psoriasis in a tertiary care hospital. J. Evolution Med. Dent. Sci. 2020;9(19):1563-1566, DOI: 10.14260/jemds/2020/341

Submission 14-01-2020,

Peer Review 12-02-2020,

Acceptance 14-02-2020,

Published 11-05-2020.

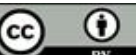




\section{BACKGROUND}

Psoriasis was derived from "PSORA" - a greek word meaning 'scale' and was first described by Robert Williams. ${ }^{[1]}$ Psoriasis is estimated to have a prevalence of around $1-2.8 \%$ in India. ${ }^{[2]}$ Psoriasis affects males two times more commonly than females and mostly in the third or fourth decade of life.[3] psoriasis is an auto-immune disease caused by multiple factors such as genetic and environmental factors. Psoriasis is a multifactorial disease with a strong genetic background. MHC region particularly HLA found in the $6 p$ chromosome has shown to be strongly associated with psoriasis, especially HLA-C*06. It has been observed that HLC-C*06 affects disease course, phenotypic features, severity, comorbidities, and treatment outcomes. This study was performed to determine the association of HLA-C with psoriasis among a south Indian ethnic population.

There are two broad clinical types of psoriasis - Pustular and non-pustular. Among these, chronic plaque psoriasis is the most common type which presents as a typical lesion which may be single or usually multiple with well-defined erythematous plaques with silvery white scales and a sharply delineated edge. Guttate type psoriasis usually occurs shortly after an acute streptococcal infections of the tonsils - affecting the children usually. Flexural inverse psoriasis appears as red shiny well demarcated plaques affecting the infra mammary, perineal and axillary regions. Palmoplantar psoriasis usually affects the palms and soles which present as scaly patches on which a fine silvery scale is seen. Psoriatic arthropathy is defined as a distinctly separate entity under seronegative spondyilo arthropathies and it is given a simple criterion for diagnosis - inflammatory arthritis, presence of psoriasis and absence of RA factor.[4]

Studies from worldwide particularly from the ethnic population of Caucasians and Han-Chinese indicate that HLA $\mathrm{C}^{*} 06$ is strongly associated with psoriasis.[5,6] There are no molecular studies showing association of HLA - C*06 with psoriasis from these Tamil speaking ethnic population. Hence this study was undertaken to determine the association of HLA-C in psoriasis patients in a south Indian ethnic population.

\section{METHODS}

This is a case control study, conducted by the Department of Transfusion Medicine, Dermatology and Human Genetics at a tertiary care teaching hospital after obtaining Institutional Ethics Committee approval (Ref: IEC-N1/11/OCT/25/62). The study was undertaken from April 2014 to March 2017. A total of 100 psoriasis patients who attended the Dermatology OP and 100 normal healthy controls who were voluntary blood donors donating at SRMC blood bank were taken up for the study. The prevalence of psoriasis is around $2 \%$ and the number of patients attending Dermatology OPD is around 5000 annually. Therefore the sample size was calculated in percentage ( $2 \%$ of 5000 is 100$)$. Newly diagnosed psoriasis patients were also included in the study. PASI (psoriasis Area Severity Index) score was calculated by the Dermatologist at the Dermatology OPD during the first visit [7]. Blood samples for HLA typing were collected after obtaining informed consent from the participants. HLA typing for Class I - A \& B was done by PCR-SSP method in the Department of Human Genetics.

Three ml blood samples were collected in sterile EDTA containers from 100 psoriasis patients and 100 healthy blood donors for HLA typing. DNA was isolated from the peripheral blood by Miller's method. DNA isolation was performed using Qualigen diagnostics as per the kit inserts provided and SOP followed. Isolated DNA was stored in $-70^{*} \mathrm{c}$ freezer in the Department of Human Genetics for HLA typing to be done.

HLA typing was performed using BAG health care $\mathrm{GmbH}$ kits (Combo kits for HLA A \& B for 100 cases and controls). SOP provided by Department of Human Genetics was followed regarding setting up of PCR thermal cycler, master mix preparation and agarose gel electrophoresis. HLA -A and HLA$B$ results were interpreted.

\section{RBC Lysis}

To the peripheral blood $40 \mu \mathrm{l}$ of RBC lysis buffer was added mixed thoroughly and incubated at room temperature for 1 hour. Samples were then centrifuged at $2000 \mathrm{rpm}$ for $20 \mathrm{~min}$.

\section{DNA Precipitation}

The resulting supernatant was transferred to a centrifuge tube and $7 \mathrm{ml}$ of isopropyl alcohol was added. The tube was mixed gently until the DNA precipitated. The precipitated DNA was then transferred to a $1.5 \mathrm{ml}$ sterile micro centrifuge tube and $500 \mu$ litre of $70 \%$ ethanol was added and spun at $2000 \mathrm{rpm}$ for $5 \mathrm{~min}$. at $4^{\circ} \mathrm{C}$. Ethanol was discarded; DNA was air dried and then dissolved in $150 \mu \mathrm{l}$ of buffer. The sample was then refrigerated at $4^{\circ} \mathrm{C}$ for complete dissolution and stored at $20^{\circ} \mathrm{C}$ till further use.

\section{Spectrophotometric Analysis}

The quality and quantity of the DNA sample was assessed by spectrophotometer. $10 \mu \mathrm{l}$ of the DNA was diluted with $990 \mu \mathrm{l}$ of TE buffer and OD (Optical Density) was read at a wavelength of $260 \mathrm{~nm}$ and $280 \mathrm{~nm}$. The concentration of the samples was determined based on the OD ratio. 1 OD corresponds to 50 $\mathrm{ng} / \mathrm{ml}$ of double stranded DNA. Samples with $260 \mathrm{~nm}$ and 280 nm OD ratio between 1.7 to 1.9 were selected for PCR.

\section{PCR (Polymerase Chain Reaction)}

Amplification of the gene was performed using specific primers under appropriate cycling conditions of denaturation, annealing and extension in a thermal cycler.

\begin{tabular}{|c|c|c|c|}
\hline Sl. No. & Reagents & $\begin{array}{c}\text { Working } \\
\text { Concentration }\end{array}$ & $\begin{array}{c}\text { Working } \\
\text { Volume }(\boldsymbol{\mu l})\end{array}$ \\
\hline 1 & Taq DNA Polymerase buffer & $1 \mathrm{x}$ & 2 \\
\hline 2 & dNTP mix & $200 \mu \mathrm{l}$ & 0.4 \\
\hline 3 & Primer forward & $50 \mathrm{pm}$ & 0.5 \\
\hline 4 & Primer reverse & $50 \mathrm{p}$ & 0.5 \\
\hline 5 & Taq DNA polymerase & 1.5 & 0.5 \\
\hline 6 & Water & - & 15.1 \\
\hline 7 & Template & $100 \mathrm{~nm}$ & 1 \\
\hline \multicolumn{3}{|c|}{ Total = } & $\mathbf{2 0} \boldsymbol{\mu l}$ \\
\hline \multicolumn{3}{|c|}{ Table. 1 : PCR - Master Mix Preparation } \\
\hline
\end{tabular}

A master mix comprising of all components except the template DNA was prepared and aliquoted in separate tubes. The template DNA was then added, the tubes were placed in the thermal cycler and subjected to the standardized PCR conditions. The PCR conditions were standardized for each gene. 


\begin{tabular}{|c|c|c|}
\hline Steps & Temperature (in ${ }^{\circ}$ C) & Time \\
\hline Initial denaturation & 94 & $5 \mathrm{~min}$ \\
\hline Denaturation & 94 & $45 \mathrm{sec}$ \\
\hline Annealing & ${ }^{*}$ Different for different genes & ${ }^{*}$ Different for different genes \\
\hline Extension & 72 & $45 \mathrm{sec}$. to 1 min \\
\hline \multicolumn{3}{|c|}{ Repeated for 30 cycles } \\
\hline Final extension & 72 & $5 \mathrm{~min}$ \\
\hline \multicolumn{3}{|c|}{ Table 2: PCR Conditions } \\
\hline
\end{tabular}

\section{PCR Confirmation}

PCR amplification was confirmed by $2 \%$ agarose gel electrophoresis. 100 bp DNA molecular weight marker was used to confirm the amplicon size.

\section{Agarose Gel Electrophoresis}

Analysis of PCR product was done by a $2 \%$ agarose gel electrophoresis. The gel was electrophoresed at $4 \mathrm{~V} / \mathrm{cm}$ and visualized in the gel documentation system. ${ }^{[8]}$

\section{PASI (psoriasis, Area, and Severity Index) Score}

This was developed by Fredricksson and Petterson in 1978. Both intensity and extent of the Psoriatic plates are calculated separately for four anatomical regions (head, trunk, upper and lower extremities) by the dermatologist. The intensity of erythema, desquamation and induration is rated on a 5 point scale with 0 - no involvement, 1- slight, 2-Moderate, 3-Severe, 4 -Very Severe characteristics. The percentage of involvement of the four anatomical regions is assigned a numerical value of 0-6 with 0 - no involvement, $1-1$ to $9 \%, 2-10$ to $29 \%, 3-30$ to $49 \%, 4-50$ to $69 \%, 5-70$ to $89 \%, 6-90$ to $100 \%$ of body surface area involvement (BSA). When calculating the PASI score four anatomical regions are calculated according to the proportion of the whole involvement of the skin. The PASI score varies from 0 to 72 . Higher score indicates severe conditions. The main advantage of PASI score is that it is the gold standard in clinical trials.[9]

\section{Statistical Analysis}

The Odds ratio (OR), $\mathrm{p}$ (probability) value, and 95\% confidence interval were the statistical tests which were studied using open epidemiology software version 3.01. This is free and open software for epidemiological statistics. Licenses are available in full text at opensource.org/license. This study was a case control study. $p$ Value of $<0.5$ was considered statistically significant. Any HLA allele having a OR of $>1$ and with a $p$ value of $<0.5$ was considered to have a strong positive association with psoriasis and similarly any HLA allele having a $\mathrm{OR}<1$ and with a $\mathrm{p}$ value of $<0.5$ was considered to have a negative association (may be protective against the disease).

\section{RESULTS}

Fourteen different HLA-C alleles were identified in this study. Of the 100 patients, 75 were male and 25 were female. HLA-C results were analysed based on onset, gender and severity. Psoriasis patients were differentiated into early and late onset if the onset of the disease was before fourth decade of life and after fourth decade of life respectively. Patients were identified as mild, moderate and severe based on (psoriasis Area Severity Index) PASI score. PASI score of $<5$ was classified as Mild, between 5 to 10 as Moderate, and $>10$ as
Severe. As an individual allele HLA-C*06 was predominantly identified being found in $52 \%$ of the patients as an individual allele. HLA-C* 07 was found in $33 \%$ of the patients. $14 \%$ of the patients had Psoriatic Arthritis also. Among the 100 controls, 92 were male and 8 were female. HLA-C* 12 was found to be the most frequent allele (35\%) in the control group. Tamil was the mother tongue of all the 200 samples included in the study.

Among the cases, HLA-C*06 had an OR of 9.75 with a pvalue of 0.0001 . It was found in 38 male and 14 female patients and HLA-C*06 was found to be associated more with late onset psoriasis and with moderate PASI score. HLA-C*07 had an OR of 2.061 with a p-value of 0.037 . It was also found to be associated more with late onset psoriasis and with moderate PASI score. Among the 33 patients, 24 were male and 9 were female.

HLA-C* 12 was found in higher frequency among the controls (35\%). It had an OR of 0.5238 with p-value of 0.0412 . This finding suggests that HLA-C*12 may have a protective role.

\begin{tabular}{|c|c|c|c|c|c|c|c|c|c|c|c|c|}
\hline \multirow{2}{*}{ 造 } & \multirow{2}{*}{ 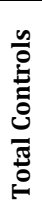 } & \multirow{2}{*}{ 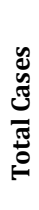 } & \multicolumn{2}{|c|}{ Onset } & \multicolumn{2}{|c|}{ Gender } & \multicolumn{3}{|c|}{$\begin{array}{c}\text { Severity } \\
\text { (PASI) }\end{array}$} & \multirow[b]{2}{*}{ ำ } & \multirow[b]{2}{*}{ 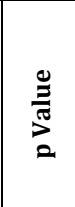 } & \multirow{2}{*}{ 월 } \\
\hline & & & 곺 & 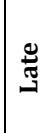 & $\frac{0}{\sum^{\pi}}$ & 荀 & $\stackrel{\text { L }}{v}$ & $\frac{}{\stackrel{1}{1}}$ & $\stackrel{\circ}{\wedge}$ & & & \\
\hline HLA - C* - 6 & 10 & 52 & 17 & 35 & 38 & 14 & 16 & 28 & 8 & 9.75 & 0.0001 & $\begin{array}{l}4.551- \\
20.89\end{array}$ \\
\hline HLA - C* - 7 & 20 & 33 & 11 & 22 & 24 & 9 & 10 & 17 & 6 & 2.061 & 0.037 & $\begin{array}{l}1.035- \\
3.749 \\
\end{array}$ \\
\hline HLA - C* 12 & 35 & 22 & \multicolumn{7}{|c|}{ Not applicable } & 0.5238 & 0.0412 & \begin{tabular}{|c|}
$0.2799-$ \\
0.9802 \\
\end{tabular} \\
\hline
\end{tabular}

\section{DISCUSSION}

HLA-C*06 allele frequency ranges from $14.1 \%$ to $59.1 \%$, but the percentage of patients with psoriasis carrying HLA-C*06 ranges from $10.5 \%$ to $77.2 \%$.[10] These studies showed that HLA-C*06 positivity in psoriasis patients in generally higher in white people than Asian population. In a meta-analysis of published studies, the Odds Ratio [OR] of HLA-C*06 in psoriasis is highest among Japanese population.[11]

A study from U.S. showed HLA-C*06 to be strongly associated with psoriasis. The study reveals that HLA-C*06 had a strong role in course of disease, severity and comorbidities associated with psoriasis.[12] A Canadian study showed HLA-C*0602 allele as the only genetic variant observed to be associated with psoriasis.[13] The role of HLA$C^{*} 06$ in psoriasis was studied in North Poland and it showed HLA-C*06 to be positively associated with early onset type and severe form of psoriasis. ${ }^{[14]}$ Shaiq et al showed in a study from Pakistan that HLA-C*06 had a strong association with psoriasis; it also mentioned a larger cohort and a denser set of HLA- markers would provide further information on HLA association with psoriasis in South Asian population.[15]

There are a few studies showing HLA association with psoriasis from Indian Population. U. Shankar Kumar showed HLA-C*0202 and HLA-C*0604 alleles to have strong association with psoriasis patients from western India.[16] One study from South India showed HLA-C*06 allele to have a positive association for susceptibility to early onset psoriasis.[17] 
The findings of this study also demonstrates the strong relationship between HLA-C*06 and psoriasis in a Tamil speaking ethnic population. The Frequency of HLA-C*06 alleles among Psoriatic patients depends on ethnicity and geographic region. For example, frequency of HLA-C*06 in Denmark is $87 \%$, Korea $76 \%$, Japan $8.2 \%$, Taiwan $6.2 \%$. The frequency of HLA-C*06 was found in around $30 \%$ Indian population.[18] In this study the frequency of HLA-C*06 was around $52 \%$, hence studies involving larger sample size needs to be done for identifying the frequency of at least HLA-C*06 if not other HLA alleles. This study also showed HLA-C*12 to have a negative association with the disease.

\section{Limitations}

This study was done on a small sample of 100 cases and 100 controls because of the high cost involved. Performing HLA-C typing on larger sample size and performing by high resolution might give results which may add more evidence on the association between HLA-C*06 and psoriasis.

\section{CONCLUSIONS}

This study has confirmed previous findings on the role of HLA$\mathrm{C}^{*} 06$ and association with psoriasis. HLA-C*06 has been shown to have a strong association with the disease in this Tamil speaking ethnic population. HLA-C*12 may have a protective role against psoriasis.

\section{REFERENCES}

[1] Christopher E, Mrowietz U. Psoriasis. Braun-Falco's Dermatology. Vol. 1. 3 $3^{\text {rd }}$ edn. Heidelberg: Springer 2010: p. 506.

[2] Kaur I, Kumar B, Sharma VK, et al. Epidemiology of psoriasis in a clinic from North India. Ind J Dermatol Venerol Leprol 1986;52(4):208-12.

[3] Gelfand JM, Weinstein R, Porter SB, et al. Prevalence and treatment of psoriasis in the United Kingdom: a population based study. Archives Dermatol 2005;141(12):1537-41.

[4] Di Megilo P, Villanova F, Nestle FO. Psoriasis. Cold Spring Harb Perspect Med 2014;4(8):a015354.
[5] Fan X, Yang S, Sun LD, et al. Comparison of clinical features of HLA-Cw6-positive and negative psoriasis patients in a Han Chinese population. Acta Derm Venereol 2007;87(4):335-40.

[6] Liu Y, Helms C, Liao W, et al. A genome-wide association study of psoriasis and psoriatic arthritis identifies new disease loci. PLoS Genet 2008;4(3):e1000041.

[7] Fredriksson T, Pettersson U. Severe psoriasis--oral therapy with a new retinoid. Dermatologica 1978;157(4):238-44.

[8] Miller SA, Dykes DD, Polesky HF. A simple salting out procedure for extracting DNA from human nucleated cells. Nucleic Acids Res 1998;16(3):1215.

[9] Naldi L, Svensson A, Diepgen T, et al. Randomized clinical trials for psoriasis 1977-2000: The EDEN survey. J Invest Dermatol 2003;120(5):738-41.

[10] Zhang XJ, Zhang AP, Yang S, et al. Association of HLA-Class I alleles with psoriasis Vulgarius in South Eastern Chinese Hans. J Dermatol Sci 2003;33(1):1-6.

[11] Mallon E, Newson R, Bunker CB. HLA-Cw6 and genetic predisposition to psoriasis: a meta-analysis of published serological studies. J Invest Dermatol 1999:113(4):693-5.

[12] Chandran V, Bull SB, Pallett FJ, et al. Human leukocyte antigen alleles and susceptibility to psoriatic arthritis. Human Immunology 2013;74(10):1333-8.

[13] Duffin KC, Chandran V, Gladman DD, et al. Genetics of psoriasis and Psoriatic arthritis: update and future direction. The Journal of Rheumatology 2008;35(7):1449-53.

[14] Szczerkowska-Dobosz A, Rebala Szczerkowska Z, et al. Correlation of HLA - $\mathrm{Cw}^{*} 06$ allele frequency with some clinical features of psoriasis vulgaris in the population of Northern Poland. J Appl Genet 2004;45(4):473-6.

[15] Shaiq PA, Stuart PE, Latif A, et al. Genetic association of psoriasis in a Pakistan population. British Journal of Dermatology 2013;169(2):406-11.

[16] Shankarkumar U. HLA-C allele associations in Mumbai psoriasis patients. Int J Hum Genet 2012;12(1):41-4.

[17] Indhumathi S, Rajappa M, Chandrashekar L, et al. The HLA $\mathrm{C}^{*} 6$ allele as a possible genetic predisposing factor to psoriasis in South Indian Tamils. Archives of Dermatological Research 2016;308(3):193-9.

[18] Chen L, Tsai TF. HLA-Cw6 and psoriasis. British Journal of Dermatology 2018;178(4):854-62. 